\title{
Progression of Myopic Maculopathy after Treatment of Choroidal Neovascularization
}

\author{
Cláudia L. Farinha ${ }^{a} \quad$ Alda S. Baltar $^{\mathrm{a}}$ Sandrina G. Nunes ${ }^{\mathrm{b}}$ João P. Figueira ${ }^{\mathrm{a}-\mathrm{c}}$ \\ Isabel A. Pires ${ }^{a, b}$ Maria L. Cachulo ${ }^{a, b}$ Rufino M. Silva ${ }^{a-c}$ \\ ${ }^{a}$ Medical Retina Unit, Ophthalmology Department, Centro Hospitalar e Universitário de Coimbra, \\ ${ }^{b}$ Association for Innovation and Biomedical Research on Light and Image and 'Faculty of Medicine, \\ University of Coimbra, Coimbra, Portugal
}

\section{Key Words}

Intravitreal ranibizumab · Macular atrophy · Myopic maculopathy progression · Photodynamic therapy · Visual prognosis

\begin{abstract}
Purpose: To evaluate the long-term progression of myopic maculopathy and functional outcome after treatment of myopic choroidal neovascularization (CNV) with photodynamic therapy (PDT) and/or intravitreal ranibizumab (IVR). Methods: Retrospective study with a cross-sectional evaluation. Eyes were assigned to 4 groups (PDT, IVR, PDT + IVR, dry myopic maculopathy) and evaluated with best-corrected visual acuity, color fundus photography and spectral-domain optical coherence tomography. Chorioretinal atrophy progression was quantified. Results: Fifty-four eyes were included with a mean follow-up of $80.6 \pm 28.0$ months. The prevalence of diffuse, patchy and macular atrophy increased during the follow-up, in contrast with tessellated fundus, lacquer cracks and active CNV. Progression of macular atrophy was significant in the 3 treatment groups $(p<0.05)$ and predictive of visual acuity. It depended on age, degree of myopia and presence of staphyloma, but not on the type of treatment. Conclusions: The long-term functional outcome of eyes with myopic CNV is more dependent on the progression of macular atrophy, and not on the type of treatment.
\end{abstract}

(c) 2014 S. Karger AG, Basel
() 2014 S. Karger AG, Basel

0030-3755/14/2314-0211\$39.50/0

\section{Introduction}

Pathological myopia is a major cause of irreversible vision loss, and is the fourth to ninth most frequent cause of blindness in the world [1-6]. Importantly, pathological myopia is the primary cause of choroidal neovascularization $(\mathrm{CNV})$ in individuals younger than 50 years $[7,8]$. Myopic maculopathy is the primary cause of vision loss associated with high myopia [9-11], and the classification made by Avila et al. [12] has been the most widely used until recently. However, it is not consensual, as stated by Hayashi et al. [10], because it is not based on the actual progression pattern and includes posterior staphyloma in the classification. Hayashi et al. [10] recently suggested a new classification system based on the progression pattern of myopic maculopathy: tessellated fundus, diffuse chorioretinal atrophy, patchy chorioretinal atrophy, lacquer cracks, active CNV and macular atrophy.

Few studies have been made in order to analyze the progression pattern of myopic maculopathy and longterm visual prognosis $[4,9,10]$. Besides, the impact of different treatments for myopic CNV on them has not been addressed so far. Until recently, photodynamic therapy (PDT) with verteporfin $\left(\right.$ Visudyne ${ }^{\circledR}$ ) was the only approved treatment for myopic CNV [7, 13, 14]; however, in the last few years several studies also reported good results with anti-vascular endothelial growth factor (VEGF) drugs, without significant complications [15-20], and the

\section{KARGER}

E-Mail karger@karger.com

www.karger.com/oph
Rufino Silva, $\mathrm{MD}, \mathrm{PhD}$

Centro de Responsabilidade Integrado de Oftalmologia do

Centro Hospitalar e Universitário de Coimbra, Praceta Mota Pinto PT-3049 Coimbra (Portugal)

E-Mail rufino.silva@oftalmologia.co.pt 
European Medicines Agency recently approved ranibizumab for the treatment of myopic CNV in Europe.

The purpose of this study is to evaluate the functional and morphological progression of myopic maculopathy on a long-term basis in eyes with myopic CNV treated with PDT, intravitreal ranibizumab (IVR), or IVR after PDT failure.

\section{Material and Methods}

A retrospective study was conducted at the Ophthalmology Department of the Centro Hospitalar e Universitário de Coimbra and at the Association for Innovation and Biomedical Research on Light and Image. Written informed consent was obtained from all subjects, and the study was conducted in accordance with the tenets of the Declaration of Helsinki and after institutional review board approval.

The medical records of consecutive patients with pathological myopia followed in our Department were examined. The inclusion criteria were: (1) highly myopic eyes, defined as having a spherical equivalent (SE) refractive error equal or more negative than $-6.00 \mathrm{dpt}$ and/or axial length (AL) equal or superior to $26.0 \mathrm{~mm}$; (2) $\mathrm{CNV}$ treated with PDT and/or IVR, without other previous treatments; (3) a minimum follow-up of 3 years if treated with IVR alone, or 5 years if treated with PDT alone or PDT and IVR; (4) contralateral highly myopic eyes that never developed $\mathrm{CNV}$ with a minimum follow-up of 3 years. The exclusion criteria were: history of amblyopia, glaucoma, uveitis, dense cataract, diabetic retinopathy, retinal vascular abnormalities, laser treatment, intravitreous injection of triamcinolone, previous vitrectomy and scleral buckling procedure.

Eyes with a history of myopic CNV meeting the inclusion criteria were assigned to 3 groups according to treatment received during the follow-up: PDT only, IVR only and PDT + IVR. The kind of treatment used for myopic CNV in the included eyes was determined as follows: after PDT approval for the treatment of myopic CNV, this was the standard treatment used in our Department, and eyes treated with PDT only were included in the PDT group; however, after IVR approval for the treatment of CNV secondary to age-related macular degeneration, new cases of myopic $\mathrm{CNV}$ were treated with IVR after an informed consent - this became the IVR group. Eyes with myopic CNV first treated with PDT but with poor response or relapsing CNV were switched to IVR during the follow-up, after informed consent, and included in the PDT + IVR group. Both treatments were never performed simultaneously during the follow-up. Fluorescein angiography and optical coherence tomography (OCT) were always performed before starting treatment for myopic CNV in all treated eyes (PDT, IVR and PDT + IVR). During the follow-up, retreatment with PDT was performed if leakage was present in fluorescein angiography. Retreatment with IVR was performed when active myopic CNV was clinically suspected and based on the presence of fluid in the OCT, best-corrected visual acuity (BCVA) loss associated with metamorphopsia and/or macular hemorrhage. Fluorescein angiography was also performed before retreatment with IVR if considered necessary. A fourth group - 'dry myopic maculopathy' - included the contralateral highly myopic eyes without history of CNV, which never required any treatment.
The patients' medical records were reviewed for data, including demographic characteristics, initial refraction, BCVA with Early Treatment of Diabetic Retinopathy Study (ETDRS) charts during follow-up, number and kind of treatments performed for myopic $\mathrm{CNV}$ and surgical treatments. Color fundus photographs were also selected for the longitudinal analysis of myopic maculopathy progression.

A cross-sectional evaluation was performed in all patients including BCVA using ETDRS charts, slitlamp examination and dilated fundus stereoscopic examination with +90 - and +60 -dpt lenses. Presence of posterior staphyloma was recorded and classification performed according to Curtin [21]. In phakic patients the manifest refraction was recorded, but it was excluded in patients who had cataract or refractive surgery during the follow-up. Measurement of axial length was performed with Biograph Allegro ${ }^{\circledR}$ (WaveLight AG, Erlangen, Germany) or by A-scan ultrasonography (OcuScan ${ }^{\circledR}$ RxP, Alcon, Calif., USA) if the previous failed. Color fundus photography and fundus autofluorescence (FAF; Heidelberg Engineering, Heidelberg, Germany) were performed for classification of myopic maculopathy. Spectral-domain OCT was performed with Spectralis OCT (Heidelberg Engineering).

The areas of macular, patchy and peripapillary atrophy were measured manually by a certified grader in all color fundus photographs and also in the FAFs performed during the last visit, using RetmarkerAMD ${ }^{\circledR}$ software (Critical Health SA, Portugal), a semiautomated system. This software divides the posterior pole into 10 subfields with a circular grid centered in the macula and calibrated through the edges of the papilla, identical to the ETDRS-style macular grid (which comprises 1-, 3- and 6-mm concentric circles) [22]. The central subfield 1 overlaps the central macula. Total and central subfield 1 areas were calculated for each type of atrophy in the selected examinations, so it was possible to precisely locate and measure their progression over time (fig. 1).

Classification of myopic maculopathy in the selected color fundus photographs was made by 2 of the authors (C.L.F., R.M.S.) independently, and in accordance with the progression pattern proposed by Hayashi et al. [10]. In cases of disagreement, the color fundus photographs were assessed jointly until a consensus was reached.

Statistical analyses were performed using the STATA software version 12.0 (StataCorp LP). Data was presented descriptively and analyzed statistically. First univariate analyses were performed to identify statistically significant results. To test for statistically significant differences between independent groups, the Mann-Whitney test was used, while for paired groups the Wilcoxon test was used. Correlations between parameters were tested using the Spearman correlation coefficient. A multivariate analysis was performed thereafter using regression models to identify risk factors for BCVA and macular atrophy. Quantile regression models were performed considering treatment, demographic and ophthalmological variables (first model) and considering the retinal lesions (second model).

\section{Results}

\section{Patient Demographic Data}

Fifty-four eyes of 30 patients ( 20 females and 10 males) were included. In some patients only 1 eye met the inclu- 
Fig. 1. Measurement of the areas of atrophy in color fundus photographs (a) and FAFs (b).
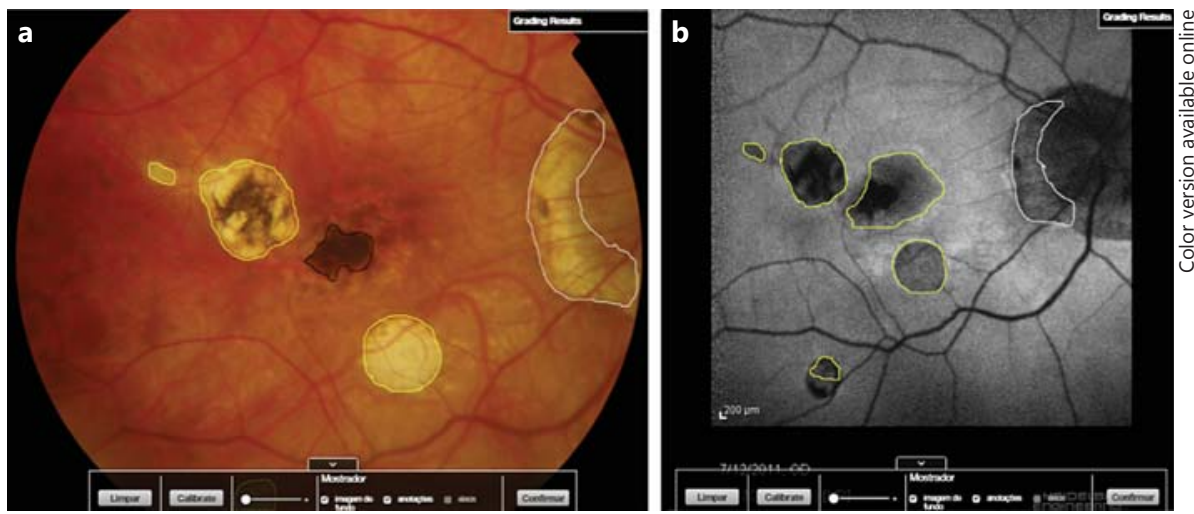

Table 1. Summary of clinical data of all eyes and by group

\begin{tabular}{|c|c|c|c|c|c|}
\hline & All eyes & PDT & IVR & $\mathrm{PDT}+\mathrm{IVR}$ & Dry maculopathy \\
\hline \multicolumn{6}{|l|}{ Age, years } \\
\hline Initial & $49.1 \pm 14.9$ & $50.3 \pm 12.1$ & $53.8 \pm 9.8$ & $45.5 \pm 19.9$ & $47.8 \pm 15.6$ \\
\hline Final & $55.4 \pm 15.2$ & $58.5 \pm 12.2$ & $57.4 \pm 9.4$ & $52.1 \pm 20.3$ & $54.0 \pm 16.3$ \\
\hline \multirow[t]{2}{*}{ Follow-up, months } & $80.6 \pm 28.0$ & $102.7 \pm 13.4$ & $43.9 \pm 4.7$ & $85.7 \pm 21.1$ & $78.5 \pm 28.6$ \\
\hline & & & & $\begin{array}{l}\text { PDT for } 43.7 \pm 31.5 \\
\text { IVR for } 42.1 \pm 11.9\end{array}$ & \\
\hline \multicolumn{6}{|l|}{ BCVA, letters } \\
\hline Initial & $55.0 \pm 21.9$ & $43.7 \pm 22.9$ & $60.1 \pm 6.3$ & $59.7 \pm 15.2$ & $58.5 \pm 28.7$ \\
\hline 36-month follow-up/before IVR & - & $51.5 \pm 24.3$ & - & $50.8 \pm 14.4$ & $61.1 \pm 28.5$ \\
\hline Final & $56.4 \pm 24.1$ & $45.5 \pm 25.7$ & $60.5 \pm 20.1$ & $58.8 \pm 18.6$ & $62.3 \pm 27.3$ \\
\hline \multicolumn{6}{|l|}{ SE, dpt } \\
\hline Initial & $-14.3 \pm 5.0$ & $-16.3 \pm 6.6$ & $-16.4 \pm 5.3$ & $-13.9 \pm 3.7$ & $-11.8 \pm 4.0$ \\
\hline Final & $-15.3 \pm 5.4$ & $-17.2 \pm 6.6$ & $-14.1 \pm 5.0$ & $-15.8 \pm 4.8$ & $-13.0 \pm 4.2$ \\
\hline $\mathrm{AL}$ at final visit, $\mathrm{mm}$ & $29.8 \pm 2.1$ & $30.7 \pm 1.9$ & $29.7 \pm 2.3$ & $29.9 \pm 2.3$ & $29.3 \pm 1.9$ \\
\hline
\end{tabular}

Data are shown as means \pm standard deviation.

sion criteria. Fifteen eyes $(27.8 \%)$ were included in the PDT group, 10 eyes (18.5\%) in the IVR group, 13 eyes (24.1\%) in the PDT + IVR group and 16 eyes $(29.6 \%)$ in the dry maculopathy group. Initial and final mean age, refractive error, axial length at the last visit, BCVA evolution and follow-up of all eyes and by group are presented in table 1 . No statistically significant differences between the 4 groups were found with respect to the initial and final age, refractive error or axial length $(p>0.05)$.

The mean number of PDT and IVR treatments performed in each group and the largest mean spot used in PDT are presented in table 2 . Ten eyes (19\%) were pseudophakic ( 2 in the PDT group, 3 in the IVR group, 2 in the PDT + IVR group and 3 in the dry maculopathy group), and 2 eyes (4\%) were aphakic ( 1 eye both in the PDT and dry maculopathy groups). Four eyes (7\%) of 2

Myopic CNV and Progression of Myopic Maculopathy patients were submitted previously to refractive surgery ( 2 in the PDT + IVR group, 1 in the IVR group and 1 in the dry maculopathy group).

\section{Characterization and Progression of Myopic \\ Maculopathy}

The progression patterns found in the 4 groups during follow-up are presented in figure 2 . The prevalence of diffuse and patchy atrophy increased during the follow-up, but the rates of tessellated fundus and lacquer cracks were lower and tended to decrease or remain constant. The exception to this pattern was the IVR group. The presence of active CNV decreased in all treatment groups with a reciprocal increase in macular atrophy throughout the follow-up. The majority of treated eyes (89\%) presented with subfoveal CNV at the beginning of the follow-up, 5 


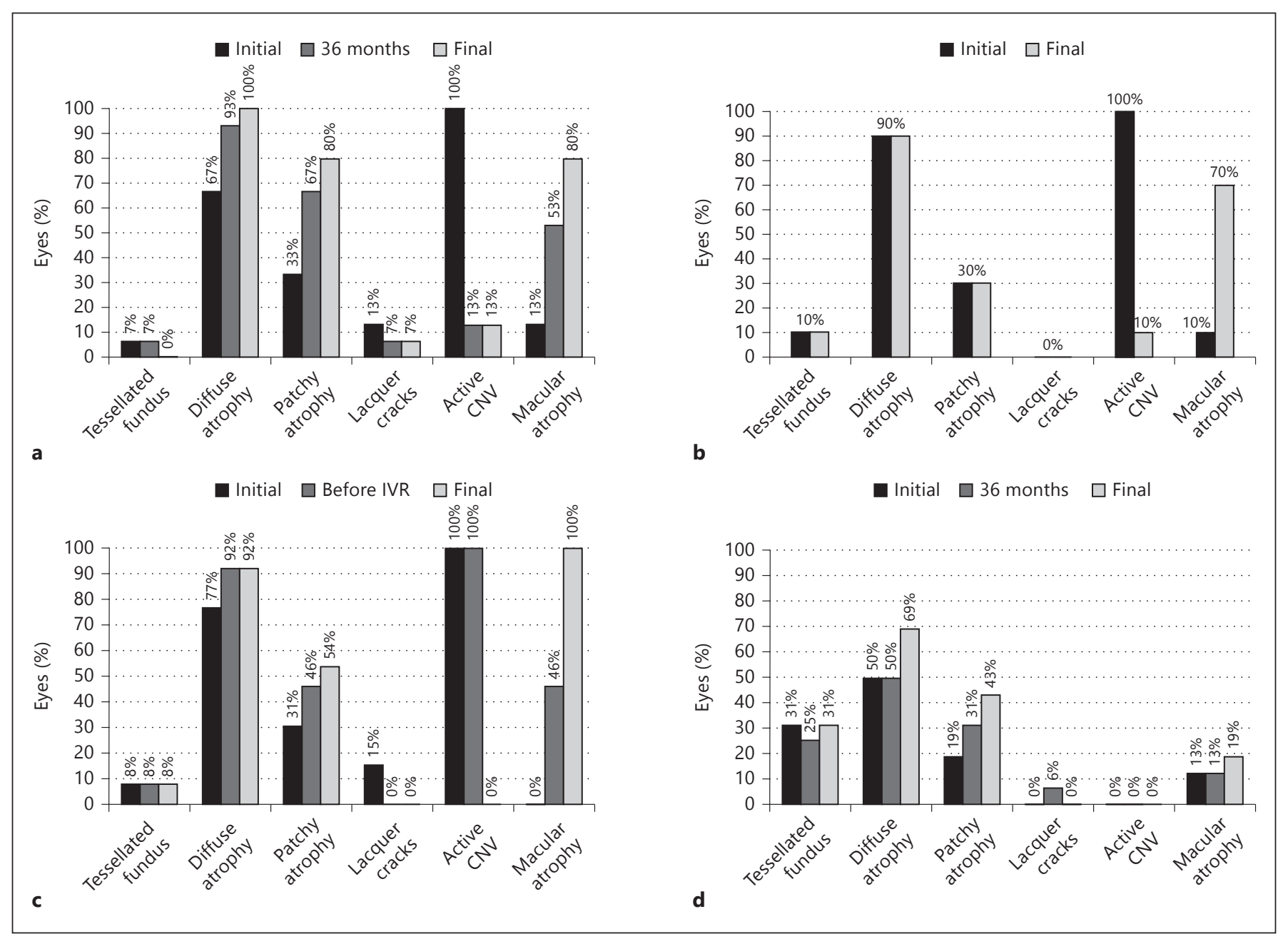

Fig. 2. Progression of myopic maculopathy in the 4 groups. a PDT. b IVR. c PDT + IVR. d Dry myopic maculopathy.

Table 2. Number of treatments performed in each group and largest mean spot used in the groups treated with PDT

\begin{tabular}{|c|c|c|c|}
\hline & PDT & IVR & $\mathrm{PDT}+\mathrm{IVR}$ \\
\hline Number of treatments with IVR & - & $7.7 \pm 4.8$ & $5.9 \pm 2.6$ \\
\hline Number of treatments with PDT & $4.1 \pm 3.0$ & - & $3.5 \pm 2.1$ \\
\hline Largest spot used in PDT, $\mu \mathrm{m}$ & $2,866.7 \pm 1,090.7$ & - & $3,180.8 \pm 848.4$ \\
\hline
\end{tabular}

Data are shown as means \pm standard deviation.

eyes (9\%) had juxtafoveal CNV, and only 1 eye (2\%) had extrafoveal CNV. Of the 30 patients included in the study, with a mean follow-up of 6.7 years, 10 developed CNV in the contralateral eye. This corresponds to a bilaterality rate of $33 \%$.
A posterior staphyloma was identified by binocular stereoscopic fundus examination in 29 eyes $(54 \%)$ in the last visit. Staphylomas type I $(\mathrm{n}=12 ; 22 \%)$ and type II $(\mathrm{n}=$ $10 ; 19 \%)$ were the most common, followed by type IV $(\mathrm{n}=3 ; 6 \%), \mathrm{V}(\mathrm{n}=3 ; 6 \%)$ and IX $(\mathrm{n}=1 ; 2 \%)$. 


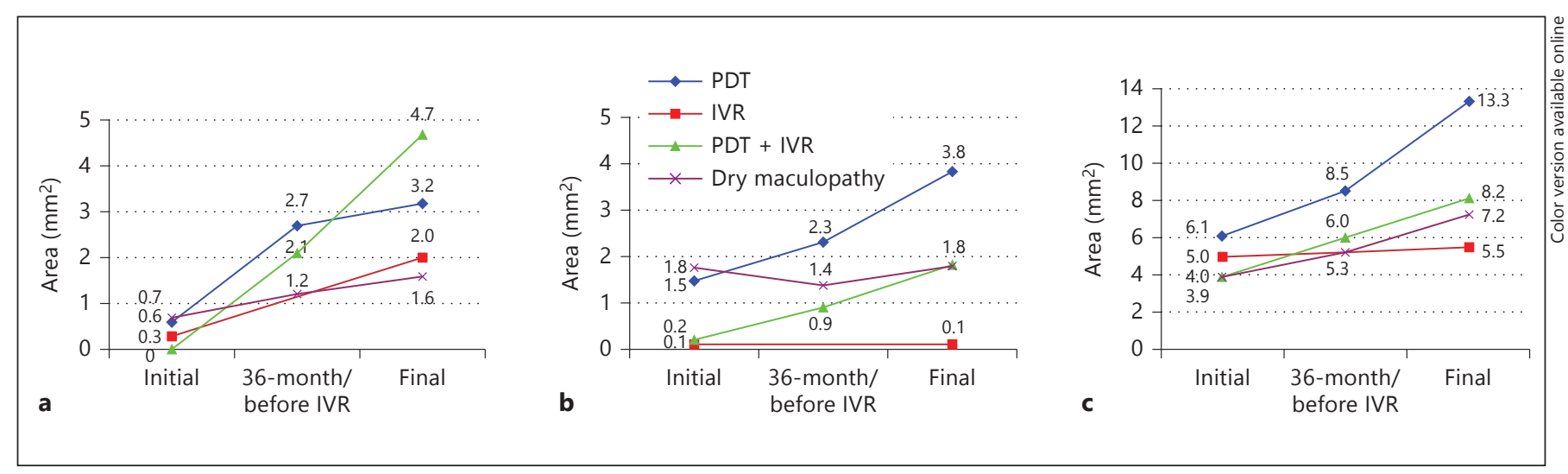

Fig. 3. Progression of the total areas of macular (a), patchy (b) and peripapillary (c) atrophy measured in the selected color fundus photographs, by group. Note: the areas are presented in square millimeters.

Table 3. Progression of the areas of myopic chorioretinal atrophy in the selected color fundus photographs, by group

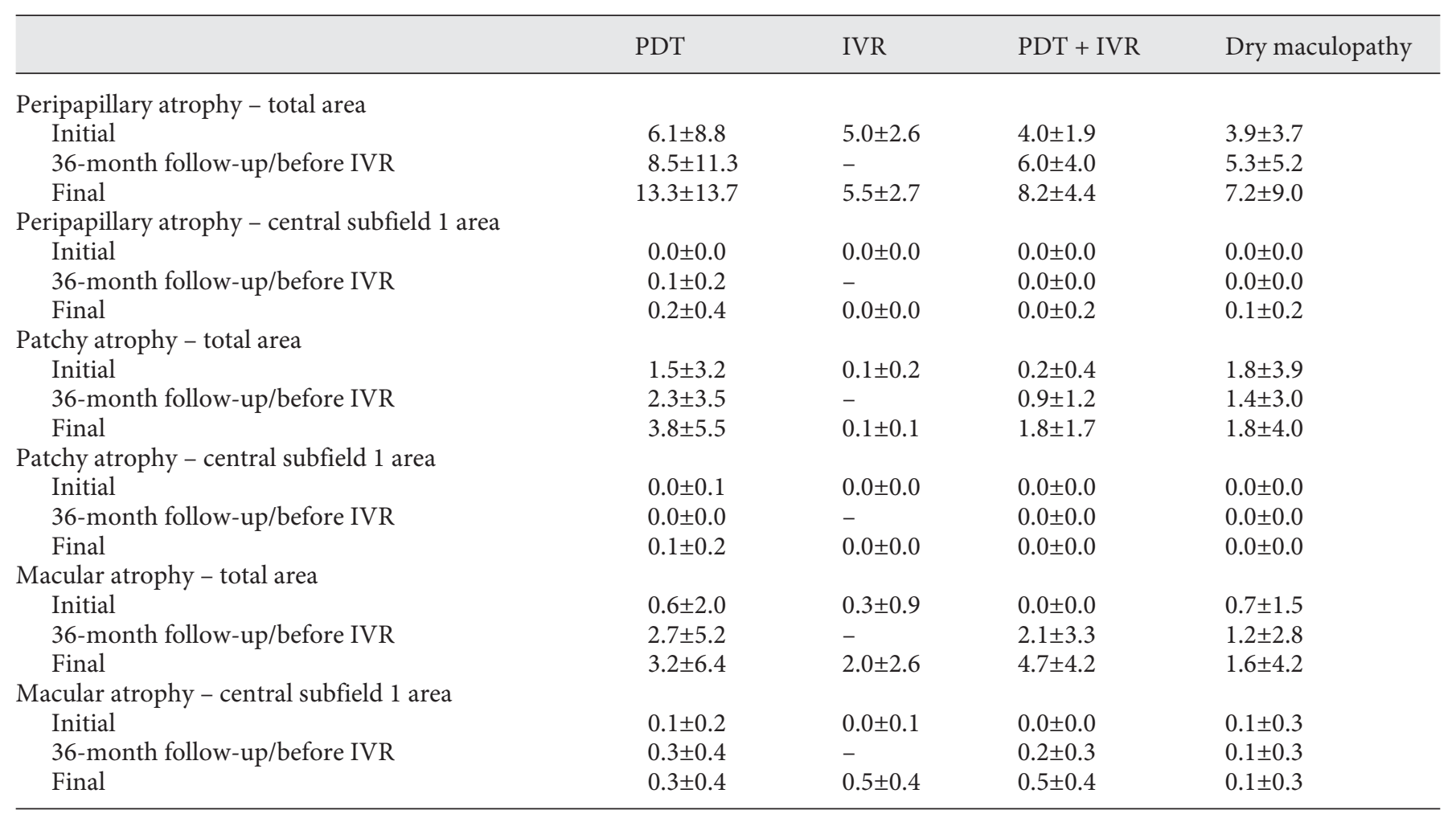

The areas are presented in square millimeters, data are shown as means \pm standard deviation.

The main associated features found on OCT in the last visit were: epiretinal membrane in $17 \%(\mathrm{n}=9)$ of all eyes, vitreomacular traction in $15 \%(\mathrm{n}=8)$ and myopic schisis in $11 \%(\mathrm{n}=6)$. Myopic schisis affecting the fovea was found only in 1 eye (PDT group).

Myopic CNV and Progression of Myopic Maculopathy

\section{Progression of Chorioretinal Atrophy}

The progression of the total and central areas of each type of chorioretinal atrophy in the color fundus photographs is presented in table 3 and figure 3 . Progression of the total and central areas of macular atrophy was statisti- 
Table 4. Areas of hypoautofluorescence in the FAFs performed during the last visit

\begin{tabular}{|c|c|c|c|c|}
\hline Final FAF & PDT & IVR & PDT + IVR & Dry maculopathy \\
\hline Total area of hypoautofluorescence from peripapillary atrophy & $8.5 \pm 8.5$ & $5.5 \pm 4.0$ & $7.0 \pm 6.3$ & $3.7 \pm 3.5$ \\
\hline Macular and patchy atrophy in total area & $5.3 \pm 7.3$ & $7.6 \pm 8.5$ & $5.8 \pm 5.2$ & $2.6 \pm 5.9$ \\
\hline
\end{tabular}

The areas are presented in square millimeters; data are shown as means \pm standard deviation.

cally significant in the 3 treatment groups during the follow-up ( $\mathrm{p}<0.05)$, but not in the dry maculopathy group. No statistically significant differences were found between the 3 treatment groups for the initial and final areas of macular atrophy (both total and central). However, a statistically significant difference was found between the dry maculopathy group and the 3 treatment groups for the final central area of macular atrophy $(\mathrm{p}<0.05)$.

The presence of macular atrophy $(\mathrm{p}=0.023)$ and the presence of CNV $(p=0.017)$ at the initial visit correlated with the final total area of macular atrophy.

The hypoautofluorescent areas corresponding to chorioretinal atrophy measured in the final FAF images were separated in areas of peripapillary atrophy and areas of macular plus patchy atrophy (table 4). A moderate correlation was found between the hypoautofluorescent areas of macular and patchy atrophy measured in the FAFs and the areas of macular and patchy atrophy measured in the color fundus photographs, at the last visit $(\mathrm{r}=0.574, \mathrm{p}<0.001)$. Also, a high correlation was found between the areas of peripapillary atrophy measured in the FAFs and the areas of peripapillary atrophy measured in the color fundus photographs $(r=0.815$, $\mathrm{p}<0.001)$.

\section{Evolution of BCVA}

Progression of BCVA from the initial evaluation to the final visit was not statistically significant in the 4 groups ( $p>0.05)$. The initial BCVA was significantly different between the PDT group and PDT + IVR group $(\mathrm{p}=0.036)$ and between the PDT group and dry maculopathy group $(\mathrm{p}=0.033)$. As for the final BCVA there were no statistically significant differences between the 4 groups ( $\mathrm{p}>0.05$; table 1 ). Considering all eyes, a positive moderate correlation was found between final BCVA and initial and final SE, meaning that less myopic eyes had a better final visual acuity $(\mathrm{r}=0.437, \mathrm{p}=0.012$, and $\mathrm{r}=0.458, \mathrm{p}=0.006$, respectively). Final BCVA was also inversely correlated with total and central areas of macular atrophy, both at the initial and final visits $(\mathrm{p}<0.01)$.

\section{Multivariate Regression Analysis}

Regression analysis was performed to identify the predictive variables for the final area of macular atrophy and for the final BCVA.

For the final total area of macular atrophy analysis, the following variables were explored: initial and final age, time of follow-up, AL, SE, presence of staphyloma, type of myopic maculopathy, area of macular and patchy atrophy, and type and number of treatments used for myopic CNV (PDT, IVR and PDT + IVR). The following showed a predictive value for a larger final area of macular atrophy: greater initial age $(p=0.010)$ and final AL $(p=0.014)$, presence of staphyloma $(\mathrm{p}<0.001)$ and a larger area of central atrophy in the initial evaluation $(p=0.011)$. Less myopic initial refraction was predictive of smaller areas $(\mathrm{p}=0.006)$. The other variables had no predictive value for macular atrophy.

For the final BCVA analysis we considered the following variables: initial and final age, follow-up, AL, SE, presence of staphyloma, type of myopic maculopathy, area of macular and patchy atrophy (total and central), and type and number of treatments used for myopic CNV. We found that only the presence and a larger total area of macular atrophy in the final visit were predictive of worse final BCVA $(\mathrm{p}<0.001, \mathrm{p}=0.008)$.

\section{Discussion}

We analyzed the long-term progression of myopic maculopathy and functional outcome in eyes treated for myopic CNV and in eyes without CNV. No significant differences in morphological and functional outcomes were found, on a long-term basis, for 3 different therapeutic approaches, PDT, IVR and PDT + IVR, and the morphological changes in treated eyes were more likely to be related with the natural progression of the myopic maculopathy than with the kind of treatment performed.

The analysis of myopic maculopathy progression was performed in a manner similar to Hayashi et al. [10], but 


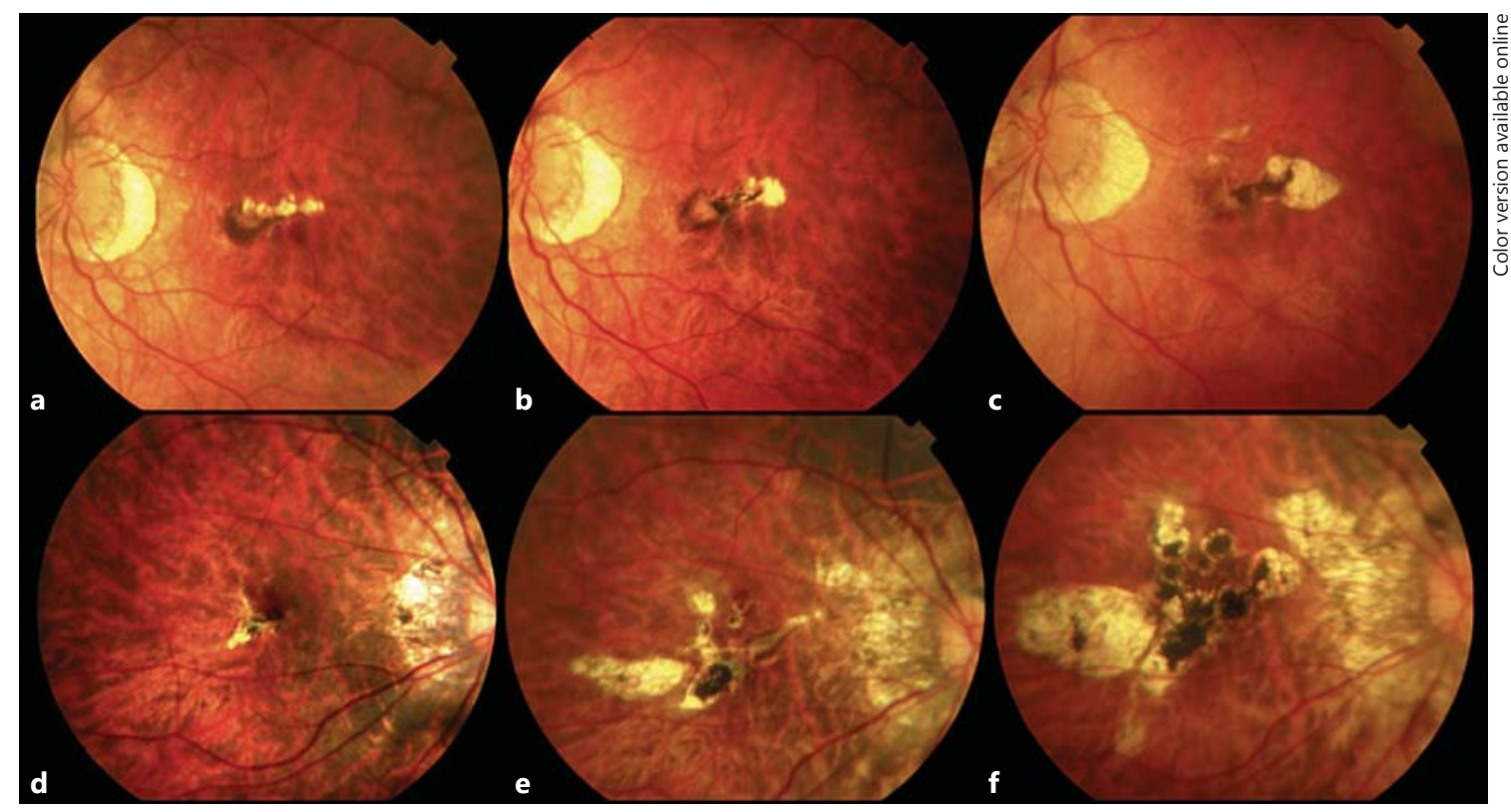

Fig. 4. a-c PDT group - 58-year-old woman, OS, initial BCVA of 59 letters and final of 77 letters, $-11.0 \mathrm{dpt}$. a Lacquer crack with CNV before treatment. b 36 months later macular atrophy developed around the regressed CNV, and patchy atrophy arose from the temporal side. c Enlargement of patchy atrophy (77 months). d-f PDT + IVR group - 39-year-old woman, OD, initial BCVA of

we also evaluated the progression in eyes treated with PDT and/or IVR for myopic CNV. In the PDT group, with a mean follow-up of 8.5 years, the tessellated fundus prevalence was very low, and the prevalence of diffuse and patchy atrophy was higher and increased until the final visit. This is probably related to the advanced stage of progression of these eyes, which already developed $\mathrm{CNV}$, and is in line with the progression patterns described by Hayashi et al. [10]. Some lacquer cracks also evolved into patchy atrophy, which explains the decrease in the first and again the increase in the latter, and confirms that lacquer cracks develop at an earlier stage than severer maculopathies [10] (fig. 4). Macular atrophy increased during the follow-up affecting $80 \%$ of the PDTtreated eyes at the final visit. This was somehow expected because macular atrophy is the most common complication of CNV. However, it was inferior to the rate of $90.1 \%$ described by Hayashi et al. [10] in nontreated myopic CNV eyes with a mean follow-up of 11.8 years.

The progression pattern found in the PDT + IVR group, which has a mean follow-up of 7.2 years, is similar to that of the PDT group. The prevalence of macular atrophy increased substantially until the final visit, even after switching to IVR, and affected all eyes in the final vis-

Myopic CNV and Progression of Myopic Maculopathy
40 letters and final of 27 letters; $-17.0 \mathrm{dpt}$. d Patchy atrophy and CNV before treatment. e After PDT treatment, at 42 months, an active $\mathrm{CNV}$ was again detected, and the number and area of patchy lesions increased; treatment was switched to IVR at this point. f Progression of the atrophic lesions (94 months).

it (fig. 4). This is important as the follow-up in the PDT + IVR group is inferior compared to the PDT group. This difference could be related to the persistence of active $\mathrm{CNV}$ in the PDT + IVR group, reflected by the need of more retreatments. Persistent disease could therefore lead to a superior chance of developing macular atrophy.

In the IVR group, with a mean follow-up of 3.7 years, the progression pattern was different. Tessellated fundus, diffuse and patchy atrophy were found with a similar distribution, but they remained constant until the final visit. Macular atrophy however increased, affecting $70 \%$ of the eyes at the final visit. Despite the inferior follow-up, the prevalence of macular atrophy in the end was similar to that of the PDT group, and if we compare the rate after a similar period of time, we find that in the PDT group it was 53\% after 3 years of follow-up. Calvo-Gonzalez et al. [19] also found that after 2 years of treatment with IVR for myopic CNV, 70.2\% of the eyes developed atrophy around the regressed CNV, and more recently, Oishi et al. [23] also reported development or enlargement of chorioretinal atrophy in $72.7 \%$ of the eyes, 4 years after treatment with bevacizumab. Moreover, Hayashi et al. [7] reported that after 3 years of natural progression of CNV $74.1 \%$ of untreated eyes developed macular atrophy, and after 5 
years the prevalence was $96.3 \%$. Therefore, the assertion of some authors that PDT leads to a superior development of macular atrophy compared to the natural evolution or treatment with anti-VEGF is questionable [24-26].

In the dry maculopathy group the prevalence of tessellated fundus was superior to treatment groups, and diffuse and patchy atrophy were considerably lower. These eyes, which never developed CNV, are probably in an earlier stage of myopic maculopathy. Although not statistically significant, they were less myopic compared to the treatment groups, and they were younger compared to the PDT and IVR groups. The relationship between these factors and the progression of myopic maculopathy was already shown by Hayashi et al. [10]. In the final assessment the rate of macular atrophy was much lower compared to treated eyes, which is in accordance with the natural history of the disease for eyes that never developed CNV [10].

In eyes treated for CNV the area of macular atrophy increased significantly until the final evaluation. This confirms previous reports that chorioretinal atrophy can develop long after CNV has regressed not only in nontreated eyes, but also in eyes treated with PDT and/or anti-VEGF $[7,27,28]$. Therefore, more studies with longterm follow-up are needed to determine the real effectiveness of different treatments for myopic CNV.

Multivariate regression analysis showed that age, degree of myopia, presence of staphyloma and a greater baseline central area of macular atrophy were predictive of greater areas of macular atrophy in the long term. According to some authors PDT could contribute to the development of macular atrophy [24, 26, 27]. However, these studies had a short follow-up, ranging from 12 to 24 months only. In a more recent study, and after the observation of long-term results, Hayashi et al. [7] state that in fact PDT apparently does not facilitate the development of macular atrophy compared to natural evolution, but treatment with intravitreal injection of bevacizumab could lead to a lower degree of macular atrophy, by preventing its appearance. Baba et al. [27] and Ruiz-Moreno and Montero [20] suggested the same. However, the latter also explained that the appearance and degree of atrophy might just be the result of the natural history of the disease. Oishi et al. [23] also state that progression of atrophy is often an inevitable consequence in the long-term follow-up of treated myopic CNV cases, but this is probably due to the natural history. Our results are more in line with this hypothesis, as the final area of macular atrophy was not significantly different between treatment groups in the long term.
Final BCVA was not significantly different between the 3 treatment groups either. This can be explained by the small sample size of the groups and the retrospective nature of the study, but the extended follow-up, somehow, strengthens these results. They appear to be in contradiction with previous reports of superior functional outcomes in eyes treated with anti-VEGF compared to eyes treated with PDT or combination treatment. However, those studies compared the functional outcome at the 1- and 2-year follow-up, and instead we evaluated it in eyes with a minimum follow-up of 3 years and a mean follow-up of 6.7 years $[25,29-31]$. The visual acuity stabilization observed in the 3 treatment groups could be the result of the development and progression of macular atrophy around the regressed CNV. This would limit the initial benefits of the treatment in visual acuity so that with a longer follow-up, as is the case of this study, the potential superiority of one treatment over the other is lost. This hypothesis was already raised in some studies $[14,20]$. A recent study reported a significant visual acuity gain 36 months after treatment of myopic CNV with ranibizumab [32], and another reported a significant gain at 48 months of follow-up, after treatment with both ranibizumab and bevacizumab [33], but in these studies, some subjects had previously been treated with PDT. Plus, el Matri et al. [25] reported that 12 months after starting treatment with bevacizumab, the gain in BCVA was no longer significant. Calvo-Gonzalez et al. [19] and Ruiz-Moreno and Montero [20], in a previous study, also found that after 2 years the gain in BCVA with anti-VEGF therapy was no longer significant. In their opinion, the decreased visual acuity during or after the second year was probably just part of the myopic degeneration. Oishi et al. [23] also reported absence of a significant gain in BCVA by the 4-year follow-up examination after treatment with bevacizumab, and he and his associates state that development and enlargement of chorioretinal atrophy frequently occurred and affected visual improvement in the long term. Thus, long-term prognosis of anti-VEGF therapy alone, especially for treatment-naïve myopic CNV, is still unclear [23].

Considering the different phenotypes of myopic maculopathy, only macular atrophy correlated with the worst visual prognosis in univariate and multivariate regression analysis. This was also found by Hayashi et al. [10] in their natural history study of pathological myopia. Final visual acuity also correlated with the initial and final areas of macular atrophy, and the final area was a negative predictor of visual outcome. Macular atrophy increased over time; however, we did not find a 
relation between time of follow-up and BCVA. Visual acuity is probably influenced by the morphological status of the macula, changing over time, but not by time per se. Location of the atrophy also seems to be important, since not only the total area of macular atrophy, but also the area of atrophy located in the central subfield correlated with final BCVA, in univariate analysis. Central location was perhaps not identified as a predictor in multivariate analysis because almost all eyes had subfoveal or juxtafoveal CNV and therefore compromise of the central macula. The predictive value of the total area of macular atrophy in BCVA represents a more pronounced degree of dysfunction of the macula with increasing atrophy. The presence and the area of macular atrophy are therefore a main predictor of visual prognosis in highly myopic eyes and may be responsible for the progressive reduction of treatment efficacy in the long term $[7,23,27]$.

There are important limitations to this study. Besides its retrospective design, the main limitation is the small number of eyes included. Although the overall group was not small, the subgroups were, the follow-up was different, and there is no true control group. However, the extended follow-up strengthens and may explain the results, as for the first time, the long-term functional and morphological outcomes in highly myopic eyes without $\mathrm{CNV}$ and in eyes with myopic CNV treated with PDT and/or IVR were evaluated.

In conclusion, our study showed no statistically significant differences in morphological and functional outcomes on a long-term basis for 3 different therapeutic approaches: PDT, IVR and PDT + IVR. Morphological and functional changes in the long term are more likely to be related with the natural progression of treated myopic $\mathrm{CNV}$ than with the kind of treatment performed.

\section{Disclosure Statement}

The authors have no proprietary or financial interest.

\section{References}

1 Krumpaszky HG, Lüdtke R, Mickler A, Klauss V, Selbmann HK: Blindness incidence in Germany. A population-based study from Württemberg-Hohenzollern. Ophthalmologica 1999;213:176-182.

2 Cotter SA, Varma R, Ying-Lai M, Azen SP, Klein R; Los Angeles Latino Eye Study Group: Causes of low vision and blindness in adult Latinos. The Los Angeles Latino Eye Study. Ophthalmology 2006;113:1574-1582.

3 Buch $\mathrm{H}$, Vinding T, Nielsen NV: Prevalence and causes of visual impairment according to World Health Organization and United States criteria in an aged, urban Scandinavian population: the Copenhagen City Eye Study. Ophthalmology 2001;108:2347-2357.

4 Vongphanit J, Mitchell P, Wang JJ: Prevalence and progression of myopic retinopathy in an older population. Ophthalmology 2002; 109:704-711.

5 Xu L, Wang Y, Li Y, Wang Y, Cui T, Li J, Jonas JB: Causes of blindness and visual impairment in urban and rural areas in Beijing: the Beijing Eye Study. Ophthalmology 2006;113: 1134.

6 Yoshida T, Ohno-Matsui K, Yasuzumi K, Kojima A, Shimada N, Futagami S, Tokoro T, Mochizuki M: Myopic choroidal neovascularization: a 10-year follow-up. Ophthalmology 2003;110:1297-1305.

7 Hayashi K, Ohno-Matsui K, Shimada N, Moriyama M, Hayashi W, Wang J, Yoshida T, Tokoro T, Mochizuki M: Long-term results of photodynamic therapy for choroidal neovas- cularization in Japanese patients with pathologic myopia. Am J Ophthalmol 2011;151: 137-147.

8 Cohen SY, Laroche A, Leguen Y, Soubrane G, Coscas GJ: Etiology of choroidal neovascularization in young patients. Ophthalmology 1996;103:1241-1244.

9 Shih YF, Ho TC, Hsiao CK, Lin LL: Visual outcomes for high myopic patients with or without myopic maculopathy: a 10 year follow up study. Br J Ophthalmol 2006;90:546550.

10 Hayashi K, Ohno-Matsui K, Shimada N, Moriyama M, Kojima A, Hayashi W, Yasuzumi K, Nagaoka N, Saka N, Yoshida T, Tokoro T, Mochizuki M: Long-term pattern of progression of myopic maculopathy: a natural history study. Ophthalmology 2010;117: 1595-1611.

11 Wang NK, Lai CC, Chu HY, Chen YP, Chen KJ, Wu WC, Yeh LK, Chuang LH, Chen TL: Classification of early dry-type myopic maculopathy with macular choroidal thickness. Am J Ophthalmol 2012;153:669-677.

12 Avila MP, Weiter JJ, Jalkh AE, Trempe CL, Pruett RC, Schepens CL: Natural history of choroidal neovascularization in degenerative myopia. Ophthalmology 1984;91:1573-1581.

13 Verteporfin in Photodynamic Therapy Study Group: Photodynamic therapy of subfoveal choroidal neovascularization in pathologic myopia with verteporfin. 1-year results of a randomized clinical trial - VIP report No 1 . Ophthalmology 2001;108:841-852.
14 Blinder KJ, Blumenkranz MS, Bressler NM, Bressler SB, Donato G, Lewis H, Lim JI, Menchini U, Miller JW, Mones JM, Potter MJ, Pournaras C, Reaves A, Rosenfeld P, Schachat AP, Schmidt-Erfurth U, Sickenberg M, Singerman LJ, Slakter JS, Strong HA, Virgili G, Williams GA: Verteporfin therapy of subfoveal choroidal neovascularization in pathologic myopia: 2-year results of a randomized clinical trial - VIP report No 3. Ophthalmology 2003;110:667-673.

15 Silva RM, Ruiz-Moreno JM, Rosa P, Carneiro A, Nascimento J, Rito LF, Cachulo ML, Carvalheira F, Murta JN: Intravitreal ranibizumab for myopic choroidal neovascularization: 12-month results. Retina 2010;30:407412.

16 Lai TY, Chan WM, Liu DT, Lam DS: Intravitreal ranibizumab for the primary treatment of choroidal neovascularization secondary to pathologic myopia. Retina 2009; 29:750-756.

17 Lalloum F, Souied EH, Bastuji-Garin S, Puche N, Querques G, Glacet-Bernard A, Coscas G, Soubrane G, Leveziel N: Intravitreal ranibizumab for choroidal neovascularization complicating pathologic myopia. Retina 2010;30: 399-406.

18 Gharbiya M, Giustolisi R, Allievi F, Fantozzi N, Mazzeo L, Scavella V, Gabrieli CB: Choroidal neovascularization in pathologic myopia: intravitreal ranibizumab versus bevacizum$\mathrm{ab}-\mathrm{a}$ randomized controlled trial. Am J Ophthalmol 2010;149:458-464. 
19 Calvo-Gonzalez C, Reche-Frutos J, Donate J, Fernandez-Perez C, Garcia-Feijoo J: Intravitreal ranibizumab for myopic choroidal neovascularization: factors predictive of visual outcome and need for retreatment. Am J Ophthalmol 2011;151:529-534.

20 Ruiz-Moreno JM, Montero JA: Intravitreal bevacizumab to treat myopic choroidal neovascularization: 2-year outcome. Graefes Arch Clin Exp Ophthalmol 2010;248:937-941.

21 Curtin BJ: The posterior staphyloma of pathologic myopia. Trans Am Ophthalmol Soc 1977;75:67-86.

22 Shin JW, Shin YU, Lee BR: Choroidal thickness and volume mapping by a six radial scan protocol on spectral-domain optical coherence tomography. Ophthalmology 2012;119: 1017-1023.

23 Oishi A, Yamashiro K, Tsujikawa A, Ooto S, Tamura H, Nakata I, Miyake M, Yoshimura $\mathrm{N}$ : Long-term effect of intravitreal injection of anti-VEGF agent for visual acuity and chorioretinal atrophy progression in myopic choroidal neovascularization. Graefes Arch Clin Exp Ophthalmol 2013;251:1-7.
24 Hayashi K, Ohno-Matsui K, Teramukai S, Shimada N, Moriyama M, Hayashi W, Yoshida T, Tokoro T, Mochizuki M: Comparison of visual outcome and regression pattern of myopic choroidal neovascularization after intravitreal bevacizumab or after photodynamic therapy. Am J Ophthalmol 2009;148:396-408.

25 El Matri L, Kort F, Chebil A, Bouraoui R, Merdassi A, Bouladi M: Intravitreal bevacizumab versus photodynamic therapy for myopic choroidal neovascularization in a NorthAfrican population. Graefes Arch Clin Exp Ophthalmol 2011;249:1287-1293.

26 Hayashi K, Ohno-Matsui K, Teramukai S, Shimada N, Moriyama M, Hara W, Yoshida T, Tokoro T, Mochizuki M: Photodynamic therapy with verteporfin for choroidal neovascularization of pathologic myopia in Japanese patients: comparison with nontreated controls. Am J Ophthalmol 2008; 145 : 518-526.

27 Baba T, Kubota-Taniai M, Kitahashi M, Okada K, Mitamura Y, Yamamoto S: Two-year comparison of photodynamic therapy and intravitreal bevacizumab for treatment of myopic choroidal neovascularisation. Br J Ophthalmol 2010;94:864-870.

28 Uemoto R, Nakasato-Sonn H, Kawagoe T, Akira M, Okada E, Mizuki N: Factors associated with enlargement of chorioretinal atrophy after intravitreal bevacizumab for myopic choroidal neovascularization. Graefes Arch Clin Exp Ophthalmol 2012;250:989-997.
29 Ikuno Y, Nagai Y, Matsuda S, Arisawa A, Sho K, Oshita T, Takahashi K, Uchihori Y, Gomi F: Two-year visual results for older Asian women treated with photodynamic therapy or bevacizumab for myopic choroidal neovascularization. Am J Ophthalmol 2010;149: 140-146.

30 Yoon JU, Byun YJ, Koh HJ: Intravitreal antiVEGF versus photodynamic therapy with verteporfin for treatment of myopic choroidal neovascularization. Retina 2010;30:418-424.

31 Wang E, Chen Y: Intravitreal anti-vascular endothelial growth factor for choroidal neovascularization secondary to pathologic myopia: systematic review and meta-analysis. Retina 2013;33:1375-1392.

32 Franqueira N, Cachulo ML, Pires I, Fonseca P, Marques I, Figueira J, Silva R: Long-term follow-up of myopic choroidal neovascularization treated with ranibizumab. Ophthalmologica 2012;227:39-44.

33 Ruiz-Moreno JM, Arias L, Montero JA, Carneiro A, Silva R: Intravitreal anti-VEGF therapy for choroidal neovascularisation secondary to pathological myopia: 4-year outcome. Br J Ophthalmol 2013;97:1447-1450. 